\title{
A Machine-Checked Proof of the Average-Case Complexity of Quicksort in Coq
}

\author{
Eelis van der Weegen` and James McKinna \\ Institute for Computing and Information Sciences \\ Radboud University Nijmegen \\ Heijendaalseweg 135, 6525 AJ Nijmegen, The Netherlands \\ eelis@eelis.net, james.mckinna@cs.ru.nl
}

\begin{abstract}
As a case-study in machine-checked reasoning about the complexity of algorithms in type theory, we describe a proof of the average-case complexity of Quicksort in Coq. The proof attempts to follow a textbook development, at the heart of which lies a technical lemma about the behaviour of the algorithm for which the original proof only gives an intuitive justification.

We introduce a general framework for algorithmic complexity in type theory, combining some existing and novel techniques: algorithms are given a shallow embedding as monadically expressed functional programs; we introduce a variety of operation-counting monads to capture worst- and average-case complexity of deterministic and nondeterministic programs, including the generalization to count in an arbitrary monoid; and we give a small theory of expectation for such non-deterministic computations, featuring both general map-fusion like results, and specific counting arguments for computing bounds.

Our formalization of the average-case complexity of Quicksort includes a fully formal treatment of the 'tricky' textbook lemma, exploiting the generality of our monadic framework to support a key step in the proof, where the expected comparison count is translated into the expected length of a recorded list of all comparisons.
\end{abstract}

\section{Introduction}

Proofs of the $O(n \log n)$ average-case complexity of Quicksort [1] are included in many textbooks on computational complexity [9, for example]. This paper documents what the authors believe to be the first fully formal machine-checked version of such a proof, developed using the Coq proof assistant 2 .

The formalisation is based on the "paper proof" in [9, which consists of three parts. The first part shows that the total number of comparisons performed by the algorithm (the usual complexity metric for sorting algorithms) can be written as a sum of expected comparison counts for individual pairs of input list elements. The second part derives from the algorithm a specific formula for

\footnotetext{
* Research carried out as part of the Radboud Master's programme in "Foundations". 
this expectation. The third and last part employs some analysis involving the harmonic series to derive the $O(n \log n)$ bound from the sum-of-expectations.

Of these three parts, only the first two involve the actual algorithm itselfthe third part is strictly numerical. While the original proof provides a thorough treatment of the third part, its treatment of the first two parts is informal in two major ways.

First, it never actually justifies anything in terms of the algorithm's formal semantics. Indeed, it does not even formally define the algorithm in the first place, relying instead on assertions which are taken to be intuitively true. While this practice is common and perfectly reasonable for paper proofs intended for human consumption, it is a luxury we can not afford ourselves.

Second, the original proof (implicitly) assumes that the input list does not contain any duplicate elements, which significantly simplifies its derivation of the formula for the expected comparison count for pairs of individual input list elements. We take care to avoid appeals to such an assumption.

The key to giving a proper formal treatment of both these aspects lies in using an appropriate representation of the algorithm, capable of capturing its computational behaviour - specifically, its use of comparisons - in a way suitable for subsequent formal reasoning. The approach we take is to consider such operation-counting as a side effect, and to use the general framework of monads for representing side-effecting computation in pure functional languages. Accordingly we use a shallow embedding, in which the algorithm, here Quicksort, is written as a monadically expressed functional program in Coq. This definition is then instantiated with refinements of operation-counting monads to make the comparison count observable.

The embedding is introduced in section 2, where we demonstrate its use by first giving a simple deterministic monadic Quicksort definition, and then instantiating it with a simple operation counting monad that lets us prove its quadratic worst-case complexity.

For the purposes of the more complex average-case theorem, we then give (in section 3) a potentially-nondeterministic monadic Quicksort definition, and compose a monad that combines operation counting with nondeterminism, supporting a formal definition of the notion of the expected comparison count, with which we state the main theorem in section 4 .

The next two sections detail the actual formalised proof. Section 5 corresponds to the first part in the original proof described above, showing how the main theorem can be split into a lemma (stated in terms of another specialized monad) giving a formula for the expected comparison count for individual pairs of input elements, and a strictly numerical part. Since we were able to fairly directly transcribe the latter from the paper proof, using the existing real number theory in the Coq standard library with few complications and additions, we omit discussion of it here and refer the interested reader to the paper proof.

Section 6 finishes the proof by proving the lemma about the expected comparison count for individual input list elements. Since this is the part where the original proof omits the most detail, and makes the assumption regarding 
duplicate elements, and where we really have to reason in detail about the behaviour of the algorithm, it is by far the most involved part of the formalisation.

Section 7 ends with conclusions and final remarks.

The Coq source files containing the entire formalisation can be downloaded from http://www. eelis.net/research/quicksort/. We used Coq version 8.2.

Related work. In his Ph.D thesis [12, Hurd presents an approach to formal analysis of probabilistic programs based on a comprehensive formalisation of measure-theoretic constructions of probability spaces, representing probabilistic programs using a state-transforming monad in which bits from an infinite supply of random bits may be consumed. He even mentions the problem of proving the average-case complexity of Quicksort, but leaves it for future work.

In [1], Audebaud and Paulin-Mohring describe a different monadic approach in which programs are interpreted directly as measures representing probability distributions. A set of axiomatic rules is defined for estimating the probability that programs interpreted this way satisfy certain properties.

Compared to these approaches, our infrastructure for reasoning about nondeterministic programs is rather less ambitious, in that we only consider finite expectation based on naïve counting probability, using a monad for nondeterminism which correctly supports weighted expectation. In particular, we do not need to reason explicitly with probability distributions.

A completely different approach to type-theoretic analysis of computational complexity is to devise a special-purpose type theory in which the types of terms include some form of complexity guarantees. Such an approach is taken in [4, for example.

\section{A Shallow Monadic Embedding}

As stated before, the key to giving a proper formal treatment of those parts of the proof for which the original contents itself with appeals to intuition, lies in the use of an appropriate representation of the algorithm. Indeed, we cannot even formally state the main theorem until we have both an algorithm definition and the means to denote its use of comparisons.

Since we are working in Coq, we already have at our disposal a full functional programming language, in the form of Coq's CIC [3. However, just writing the algorithm as an ordinary Coq function would not let us observe its use of comparisons. We can however see comparison counting as a side effect. As is well known and standard practice in functional languages such as Haskell, side effects can be represented using monads: a side-effecting function $f$ from $A$ to $B$ is represented as a function $A \rightarrow M B$ where $M$ is a type constructor encapsulating the side effects. "Identity" and "composition" for such functions are given by ret (for "return") of type $A \rightarrow M A$ and bind (infix: $»$ ) of type $M A \rightarrow(A \rightarrow M B) \rightarrow M B$ satisfying certain identities (the monad laws). For a general introduction to monadic programming and monad laws, see [5]. 
Furthermore, we use Haskell's "do-notation", declared in Coq as follows

Notation $\mathrm{x}<-\mathrm{y} ; \mathrm{z} ":=\left(\right.$ bind $\left.y\left(\lambda x:_{-} \Rightarrow z\right)\right)$

and freely use standard monadic functions such as:

liftM $: \forall(M:$ Monad $)(A B:$ Set $),(A \rightarrow B) \rightarrow(M A \rightarrow M B)$

filter $M: \forall(M:$ Monad $)(A:$ Set $),(A \rightarrow M$ bool $) \rightarrow$ list $A \rightarrow M($ list $A)$

Here, the Coq type Monad is a dependent record containing the (coercible) carrier of type Set $\rightarrow$ Set, along with the bind and ret operations, and proofs of the three monad laws.

We now express Quicksort in this style, parameterizing it on both the monad itself and on the comparison operation. A deterministic Quicksort that simply selects the head of the input list as its pivot element, and uses two simple filter passes to partition the input list, looks as follows:

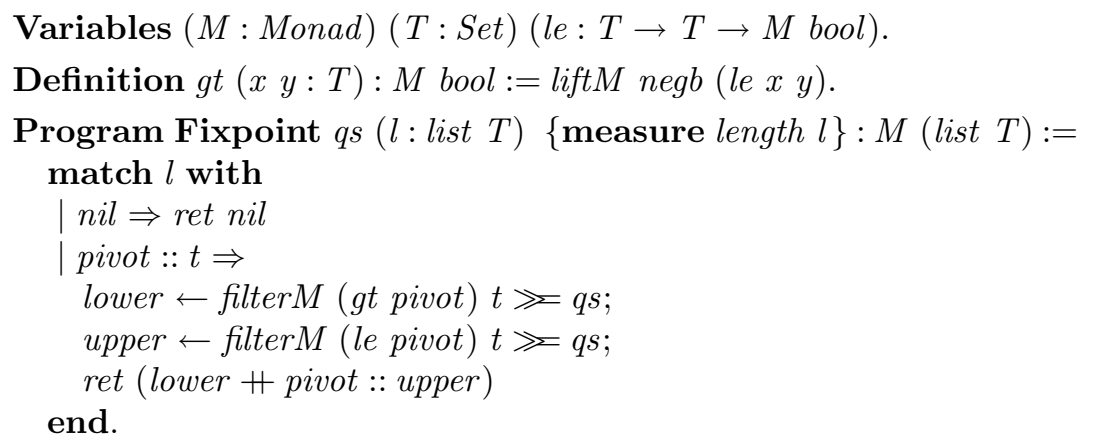

We use Coq's Program Fixpoint facility [7] to cope with Quicksort's nonstructural recursion, specifying list length as an input measure function that is separately shown to strongly decrease for each recursive call. For this definition of $q s$, these proof obligations are trivial enough for Coq to prove mostly by itself.

For recursive functions defined this way, Coq does not automatically define corresponding induction principles matching the recursive call structure. Hence, for this $q s$ definition as well as the one we will introduce in section 3 we had to define these induction principles manually. To make their use as convenient as possible, we further customized and specialized them to take advantage of specific monad properties. We will omit further discussion of these issues in this paper, and will henceforth simply say: "by induction on $q s, . . . "$

By instantiating the above definitions with the right monad, we can transparently insert comparison-counting instrumentation into the algorithm, which will prove to be sufficient to let us reason about its complexity. But before we do so, let us note that if the above definitions are instead instantiated with the identity monad and an ordinary elementwise comparison on $T$, then the monadic scaffolding melts away, and the result is equivalent to an ordinary non-instrumented, non-monadic version, suitable for extraction and correctness proofs (which are included in the formalisation for completeness). This means that while we will 
instantiate the definitions with less trivial monads to support our complexity proofs, we can take some comfort in knowing that the object of those proofs is, in a very concrete sense, the actual Quicksort algorithm (as one would write it in a functional programming language), rather than some idealized model thereof.

For reasons that will become clear in later sections, we construct the monad with which we will instantiate the above definitions using a monad transformer [8] $M M T$ (for "monoid monad transformer"), which piggybacks a monoid onto an existing monad by pairing.

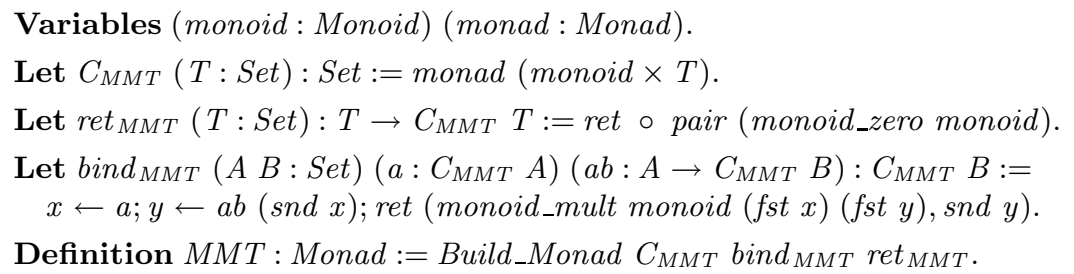

(In the interest of brevity, we omit proofs of the monad laws for $M M T$ and all other monads defined in this paper. These proofs can all be found in the Coq code.)

We now use $M M T$ to piggyback the additive monoid structure on $\mathbb{N}$ onto the identity monad, and lift elementwise comparison into the resulting monad, which we call $S P$ (for "simply-profiled").

Definition $S P$ : Monad $:=M M T(\mathbb{N}, 0,+)$ IdMonad.

Definition $l e_{S P}(x y: \mathbb{N}): S P$ bool $:=(1$, le $x y)$.

When instantiated with this monad and comparison operation, qs produces the comparison count as part of its result.

Definition $q s_{S P}:=q s S P l e_{S P}$.

Eval compute in $q s_{S P}(3:: 1:: 0:: 4:: 5:: 2::$ nil $)$. $=(16,0:: 1:: 2:: 3:: 4:: 5::$ nil $)$

Defining cost and result as the first and second projection, respectively, we trivially have identities such as cost $\left(\operatorname{ret}_{S P} x\right)=0, \operatorname{cost}\left(\begin{array}{lll}l_{S P} & x & y\end{array}\right)=1$, and cost $\left(x \gg_{S P} f\right)=\operatorname{cost} x+\operatorname{cost}(f($ result $x))$. This very modest amount of machinery is sufficient for a straightforward proof of Quicksort's quadratic worst-case complexity.

Proposition. qs_worst $: \forall l$, cost $\left.\left(q s_{S P} l\right) \leqslant(\text { length } l)^{2}\right]$

Proof. The proof is by induction on qs. For $l=n i l$, we have $\operatorname{cost}\left(q s_{S P} n i l\right)=$ cost $($ ret nil $)=0 \leqslant(\text { length } l)^{2}$. For $l=h:: t$, the cost decomposes into

${ }^{1}$ We do not use big-O notation for this simple statement, as it would only obfuscate.
Big-O complexity is discussed in section 4. 


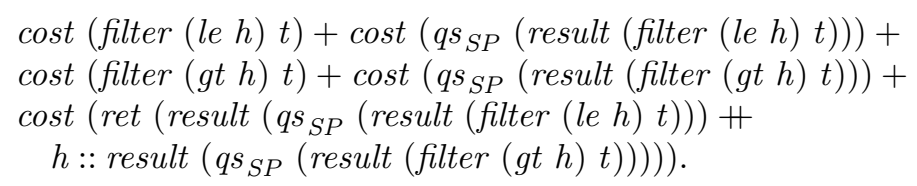

The filter costs are easily proved (by induction on $t$ ) to be length $t$ each. The cost of the final ret is 0 by definition. The induction hypothesis applies to the recursive $q s_{S P}$ calls. Furthermore, by induction on $t$, we can easily prove

$$
\text { length }(\text { result }(\text { filter }(\text { le } h) t))+\text { length }(\text { result }(\text { filter }(g t h) t)) \leqslant \text { length } t \text {, }
$$

because the two predicates filtered on are mutually exclusive. Abstracting the filter terms as $f t$ and $f t t^{\prime}$, this leaves

$$
\begin{aligned}
& \text { length } \mathrm{flt}+\text { length } \mathrm{flt} t^{\prime} \leqslant \text { length } t \rightarrow \\
& \quad \text { length } t+\left(\text { length } f(t)^{2}+\text { length } t+\left(\text { length } f\left(t^{\prime}\right)^{2}+0 \leqslant(S(\text { length } t))^{2},\right.\right.
\end{aligned}
$$

which is true by elementary arithmetic.

We now extend the technique to prepare for the average-case proof.

\section{Nondeterminism and Expected Values}

The version of Quicksort used in the average-case complexity proof in [9] differs from the one presented in the last section in two ways. This is also reflected in our formalisation.

First, the definition of $q s$ is modified to use a single three-way partition pass, instead of two calls to filter, thus avoiding the pathological quadratic behaviour which can arise when the input list does not consist of distinct elements.

Second, and more significantly, we use nondeterministic pivot selection, thus avoiding the pathological quadratic behaviour from which any deterministic pivot selection strategy inevitably suffers. While this means that we have proved our result for a subtly different presentation of Quicksort, this nevertheless follows the textbook treatment, in line with common practice.

These two modifications together greatly simplify the formalisation, because they remove the need to carefully track input distributions in order to show that 'good' inputs (for which the original deterministic version of the algorithm performs well) sufficiently outnumber 'bad' inputs (for which the original version performs poorly). They further ensure that the $O(n \log n)$ average-case bound holds not just averaged over all possible input lists, but for each individual input list as well. In particular, it means that once we prove that the bound holds for an arbitrary input, the global bound immediately follows.

This also means that for a key lemma near the end of our proof, we can use straightforward induction over the algorithm's recursive call structure, without having to show that given appropriately distributed inputs, the partition step yields lists that are again appropriately distributed. Such issues are a major technical concern in more ambitious approaches to average-case complexity analysis [10, for example] and to the analysis of probabilistic algorithms. 
The second modification is based on a new monad (again defined using $M M T$, but this time transforming a nondeterminism monad) with which the new definition can be instantiated, capturing the expected comparison count.

The first modification is relatively straightforward. Instead of calling filter $M$, which uses a two-way comparison operation producing a monadic bool, we define a function partition. It takes a three-way comparison operation producing a monadic comparison, which is an enumeration with values $L t, E q$, and $G t$. We represent the resulting partitioning by a function of type comparison $\rightarrow$ list $T$ rather than a record or tuple type containing three lists, because in the actual formalisation, this saves us from having to constantly map comparison values to corresponding record field accessors or tuple projections. This is only a matter of minor convenience; a record or tuple could have been used instead without problems.

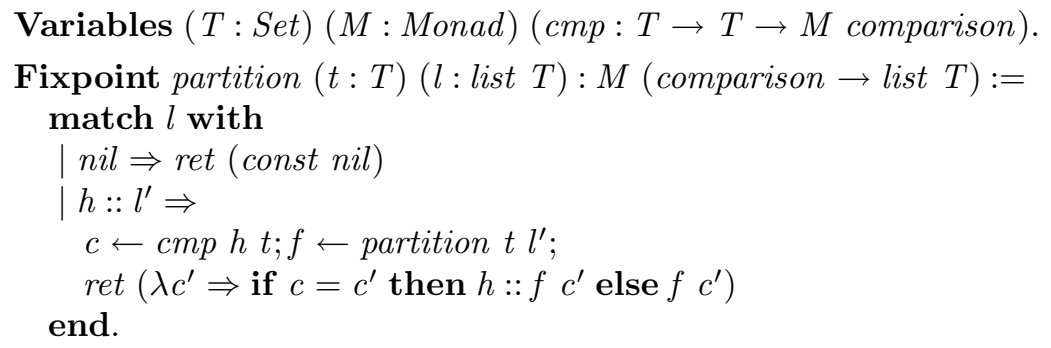

Next, we redefine $q s$ to use partition, and have it take as an additional parameter a pick operation, representing nondeterministic selection of an element of a nonempty list of choices. An ne_list $T$ is a non-empty list of $T$ 's, inductively defined in the obvious way.

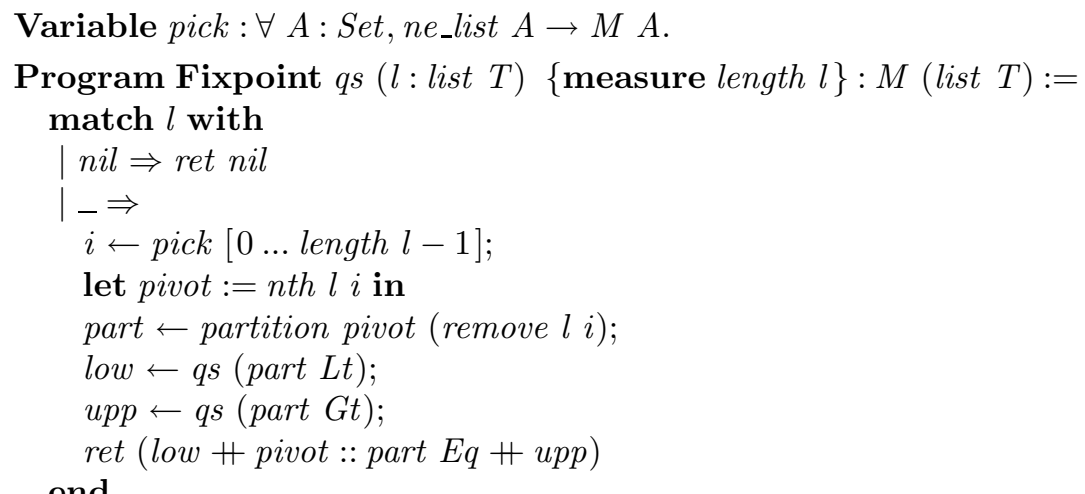

The functions $n$th and remove select and remove the $n$th element of a list, respectively.

Note that the deterministic Quicksort definition in section 2 could also have been implemented with a partition pass instead, which might well have made the worst-case proof even simpler. We chose not to do this, in order to emphasise that the properties the average-case proof demands of the algorithm rule out the naïve but familiar implementation using filter passes. 
Nondeterminism can now be emulated by instantiating these definitions with a suitable monad and pick operation. A deterministic, non-instrumented version can still be obtained, simply by using the identity monad and any deterministic pick operation, such as head or 'median-of-three' (not considered here).

Let us now consider what kind of nondeterminism monad would be suitable for reasoning about the expected value of a nondeterministic program like

$$
x \leftarrow \text { pick }[0,1] \text {; if } x=0 \text { then ret } 0 \text { else pick }[1,2] .
$$

When executed in the list monad (commonly used to emulate nondeterministic computation), this program produces $[0,1,2]$ as its list of possible outcomes. Unfortunately, the information that 0 is a more likely outcome than 1 or 2 has been lost. Such relative probabilities are critical to the notion of an expected value: the expected value of the program above is avg $[0$, avg $[1,2]]=\frac{3}{4} \neq 1=$ avg $[0,1,2]$. This makes list nondeterminism unsuitable for our purposes.

Using tree nondeterminism instead solves the problem: we introduce the type ne_tree of non-empty trees, building on ne_list:

Inductive ne_tree $(T:$ Set $):$ Set $:=$

Leaf $: T \rightarrow$ ne_tree $T$

Node: ne_list $($ ne_tree $T) \rightarrow$ ne_tree $T$.

Definition ret $_{\text {ne_tree }}\{A:$ Set $\}: A \rightarrow$ ne_tree $A:=$ Leaf .

Fixpoint bind $_{\text {ne_tree }}(A B:$ Set $)$

$\left(m: n e_{\text {_tree }} A\right)(k: A \rightarrow$ ne_tree $B):$ ne_tree $B:=$

match $m$ with

Leaf $a \Rightarrow k a$

| Node $t s \Rightarrow$ Node $\left(\right.$ ne_list.map $\left(\lambda x \Rightarrow\right.$ bind $\left.\left._{n e \_t r e e} x k\right) t s\right)$

end.

Definition $M_{n e_{-} t r e e}:$ Monad $:=$ Build_Monad ne_tree bind ne_tree $_{\text {ret }}$ re_tree $_{n e_{-}}$.

Definition pick $_{\text {ne_tree }}(T:$ Set $): n e \_l i s t ~ T \rightarrow M_{\text {ne_tree }} T$

$:=$ Node $\circ$ ne_list.map Leaf.

We use non-empty trees because we do not consider partial functions, and using potentially empty trees would complicate the definition of a tree's average value below. This is also why we used ne_list for pick.

With this monad and pick operation, the same program now produces the tree Node [Leaf 0, Node [Leaf 1, Leaf 2]], which preserves the relative probabilities. The expected value now coincides with the weighted average of these trees:

Definition ne_tree.avg : ne_tree $\mathbb{R} \rightarrow \mathbb{R}:=$ ne_tree.fold id ne_list.avg.

Relative probabilities are also the reason we use an $n$-ary choice primitive rather than a binary one, because correctly emulating (that is, without skewing the relative probabilities) an $n$-ary choice by a sequence of binary choices is only possible when $n$ is a power of two.

To denote the expected value of a discrete measure $f$ of the output of a program, we define 
Definition expec $(T: S e t)(f: T \rightarrow \mathbb{N}):$ ne_tree $T \rightarrow \mathbb{R}$ $:=$ ne_tree.avg $\circ$ ne_tree.map $f$.

Thus, given a program $P$ of type $M_{n e_{-} \text {tree }}$ (list bool), expec length $P$ denotes the expected length of the result list, if we interpret values of type $M_{n e_{-} \text {tree }} T$ as nondeterministically computed values of type $T$.

The function expec gives rise to a host of identities, such as

$$
\begin{aligned}
0 & \leq \operatorname{expec} f t \\
\text { expec }(\lambda x \Rightarrow f x+g x) t & =\operatorname{expec} f t+\text { expec } g t \\
\text { expec }((* c) \circ f) & =(* c) \circ \text { expec } f \\
(\forall x \in t \rightarrow f x \leqslant g x) \rightarrow \operatorname{expec} f t & \leq \operatorname{expec} g t \\
(\forall x \in t \rightarrow f x=c) \rightarrow \operatorname{expec} f t & =c \\
(\forall x \in t \rightarrow f x=0) \leftrightarrow \operatorname{expec} f t & =0 \\
\operatorname{expec} f(t \gg(\text { ret } \circ g)) & =\operatorname{expec}(f \circ g) t \\
\text { expec }(f \circ g) t & =\operatorname{expec} f(\text { ne_tree.map } g t)
\end{aligned}
$$

To form the monad with which we will instantiate $q s$ for the main theorem,

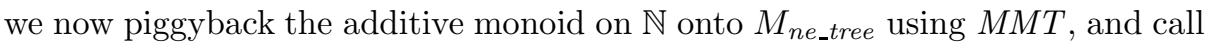
the result $N D P$ (for "nondeterministically profiled"):

Definition $M_{N D P}:$ Monad $:=\operatorname{MMT}(\mathbb{N}, 0,+) M_{n e \_t r e e}$.

Definition $c m p_{N D P}(x y: T): M_{N D P}$ bool $:=$ ret $_{n e \_t r e e}(1, c m p x y)$.

Definition $q s_{N D P}:=q s M_{N D P} c m p_{N D P}\left(\right.$ lift pick $\left.{ }_{n e_{-} \text {tree }}\right)$.

We can now denote the expected comparison count for a $q s_{N D P}$ application by expec cost $\left(q s_{N D P} l\right)$, and will use this in our statement of the main theorem in the next section.

But before we do so, we define a slight refinement of expec that specifically observes the monoid component of computations in monads formed by transforming $M_{n e_{-} t r e e}$ using $M M T$ (like $N D P$ ).

Definition monoid_expec $(m:$ Monoid $)(f: m \rightarrow \mathbb{N})\{A:$ Set $\}$

$$
:\left(M M T m M_{n_{-} \text {tree }} A\right) \rightarrow \mathbb{R}:=\operatorname{expec}(f \circ f s t) .
$$

Since $\operatorname{cost}=f_{s t}$, we have expec cost $t=$ monoid_expec $i d t$.

In addition to all the identities monoid_expec inherits from expec, it has some of its own. One identity states that if one transforms $M_{n e \_t r e e}$ using a monoid $m$, then for a monoid homomorphism $h$ from $m$ to the additive monoid on $\mathbb{N}$, monoid_expec $h$ distributes over bind, provided that the expected monoid value of the right hand side does not depend on the computed value of the left hand side:

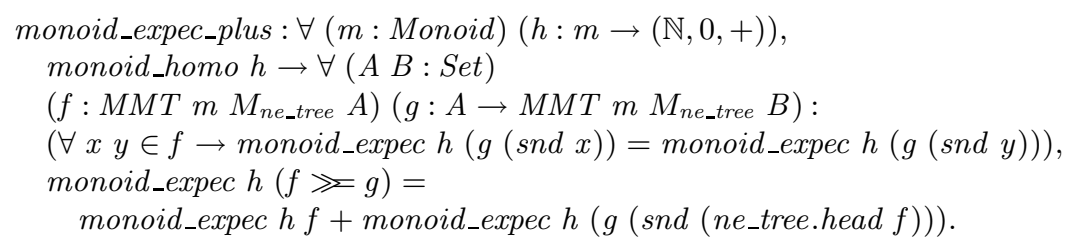


Since $i d$ is a monoid homomorphism, monoid_expec_plus applies to NDP and expec cost. In section 5 , we will use monoid_expec_plus with another monoid and homomorphism.

\section{The Statement}

The last thing needed before the main theorem can be stated, is the notion of big-O complexity. We use the standard textbook definition, except that we make explicit how we measure inputs to $f$, namely with respect to a measure function $m$ :

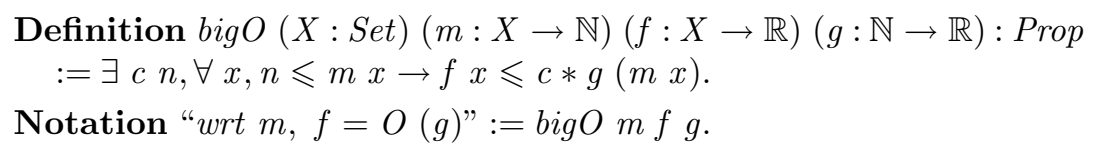

We now state the main theorem.

Theorem qs_avg: wrt length, expec cost $\circ q s_{N D P}=O\left(\lambda n \Rightarrow n * \log _{2} n\right)$.

Thanks to the property discussed at the start of the previous section, qs_avg follows as a corollary from the stronger statement

$$
\text { qs_expec_cost }: \forall l \text {, expec cost }\left(q s_{N D P} l\right) \leqslant 2 * \text { length } l *\left(1+\log _{2}(\text { length } l)\right) \text {, }
$$

the proof of which is described in the next two sections.

\section{Reduction to Pairwise Comparison Counts}

As described in the introduction, the key ingredient in the proof is a lemma giving a formula for the expected comparison count for individual pairs of input list elements, indexed a certain way. More specifically, if $X \equiv X_{I_{0}} \ldots X_{I_{n-1}}$ is the input list, with $I$ a permutation of $[0 \ldots n-1]$ such that $X_{0} \ldots X_{n}$ is sorted, then the expected comparison count for any $X_{i}$ and $X_{j}$ with $i<j$ is at most $2 /(1+j-i)$. In other words, the expected comparison count for two input list elements is bounded by a simple function of the number of list elements that separate the two in the sort order. We prove this fact in the next section, but first show how qs_expec_cost follows from it.

Combined with the observation that the total expected comparison count ought to equal the sum of the expected comparison count for each individual pair of input elements, the property described above suggests breaking up the inequality into

$$
\text { expec cost }\left(q s_{N D P} l\right) \leqslant \sum_{(i, j) \in I J} \text { ecc } i j \leqslant 2 * \text { length } l *\left(1+\log _{2}(\text { length } l)\right) \text {, }
$$

where $I J:=\{(i, j) \in[0$, length $l) \mid i<j\}$, and ecc $i j:=2 /(1+j-i)$. 
The right-hand inequality is a strictly numerical affair, requiring a bit of analysis involving the harmonic series. As stated before, this part of the proof was fairly directly transcribed from the paper proof, with few complications and additions, and so we will not discuss it.

The left inequality is the challenging one. To bring it closer to the index summation, we first write $l$ on the left-hand side as map (nth (sort $l$ )) li, where sort may be any sorting function (including qs itself), and where $l i$ is a permutation of $[0 \ldots n-1]$ such that map $(n t h($ sort $l)) l i=l$ (such an $l i$ can easily be proven to exist).

Next, we introduce a specialized monad and comparison operation that go one step further in focusing specifically on these indices.

Definition Monoid $_{U}:$ Monoid $:=($ list $(\mathbb{N} \times \mathbb{N})$, nil,+$)$.

Definition $U:$ Monad $:=$ MMT Monoid $_{U} M_{n e_{-} t r e e}$.

Definition lookup_cmp $(x y: \mathbb{N}):$ comparison $:=$ cmp (nth (sort l) $x)$ (nth (sort l) y).

Definition unordered_nat_pair $(x y: \mathbb{N}): \mathbb{N} \times \mathbb{N}:=$ if $x \leqslant y$ then $(x, y)$ else $(y, x)$.

Definition $c m p_{U}(x y: \mathbb{N}): U$ comparison $:=$ ret (unordered_nat_pair $x$ y :: nil, lookup_cmp $x$ y).

Definition $q s_{U}:$ list $\mathbb{N} \rightarrow$ list $\mathbb{N}:=q s U$ cmp $_{U}$ pick $_{U}$.

The function $q s_{U}$ operates directly on lists of indices into sort $l$. Comparison of indices is defined by comparison of the values they denote in sort $l$. Furthermore, rather than producing a grand total comparison count the way $N D P$ does, $U$ records every pair of indices compared, by using $M M T$ with Monoid $_{U}$, the free monoid over $\mathbb{N} \times \mathbb{N}$ pairs, instead of the additive monoid on $\mathbb{N}$ we used until now.

We now rewrite

$$
\begin{aligned}
& \text { expec cost }\left(q s_{N D P}(\operatorname{map}(n t h(\text { sort } l)) l i)\right) \\
& \quad=\text { monoid_expec length }\left(q s_{U} l i\right)=\text { expec }(\text { length } \circ f s t)\left(q s_{U} l i\right) .
\end{aligned}
$$

The first equality expresses that the expected number of comparisons counted by $N D P$ is equal to the expected length of the list of comparisons recorded by $U$. In the formalisation, this is a separate lemma proved by induction on $q s$. The second equality merely unfolds the definition of monoid_expec.

After rewriting with identity 1 in section 3 on page 264, the goal becomes

$$
\text { expec length (ne_tree.map fst } \left.\left(q s_{U} l i\right)\right) \leqslant \sum_{(i, j) \in I J} \text { ecc } i j \text {. }
$$

We now invoke another lemma which bounds a nondeterministically computed list's expected length by the expected number of occurrences of specific values in that list. More specifically, it states that

$$
\begin{aligned}
& \forall(X: \text { Set })(\text { fr }: X \rightarrow \mathbb{R})(q: \text { list } X)(t: \text { ne_tree }(\text { list } X)), \\
& \quad(\forall x \in q, \text { expec }(\text { count } x) t \leqslant \text { fr } x) \rightarrow \\
& \quad(\forall x \notin q, \text { expec }(\text { count } x) t=0) \rightarrow \text { expec length } t \leqslant \sum_{x \in q} f r x .
\end{aligned}
$$


We end up with two subgoals, the first of which is

$$
\forall(i, j) \notin I J, \text { expec }(\text { count }(i, j))\left(\text { ne_tree.map } f s t_{\text {- }}\left(q s_{U} l i\right)\right)=0 \text {. }
$$

Rewriting this using identity 1 from section 3 in reverse, then rewriting the expec as a monoid_expec, and then generalizing the premise, results in

$$
\forall i j l i,(i \notin l i \vee j \notin l i) \rightarrow \text { monoid_expec }(\operatorname{count}(i, j))\left(q s_{U} l i\right)=0
$$

which can be shown by induction on $q s$, although we will not do so in this paper. We will use this property again in the next section.

The second subgoal, expressed with monoid_expec, becomes

$$
\forall(i, j) \in I J, \text { monoid_expec }(\text { count }(i, j))\left(q s_{U} l i\right) \leqslant e c c i j
$$

which corresponds exactly to the property described at the beginning of this section. We prove it in the next section.

\section{Finishing the Proof}

Again, the proof of (3) is by induction on qs. But to get a better induction hypothesis, we drop the $(i, j) \in I J$ premise (because as was shown in the last section, the statement is also true if $(i, j) \notin I J)$, and add a premise saying $l i$ is a permutation of a contiguous sequence of indices.

$$
\begin{aligned}
& \forall i j, i<j \rightarrow \forall(l i: \text { list } \mathbb{N})(b: \mathbb{N}), \text { Permutation }[b \ldots b+\text { length } l i-1] l i \rightarrow \\
& \quad \text { monoid_expec }(\text { count }(i, j))\left(q s_{U} \text { li }\right) \leqslant \text { ecc } i j .
\end{aligned}
$$

In the base case, $l i$ is $n i l$, and the left-hand side of the inequality reduces to 0. In the recursive case, $q s$ unfolds:

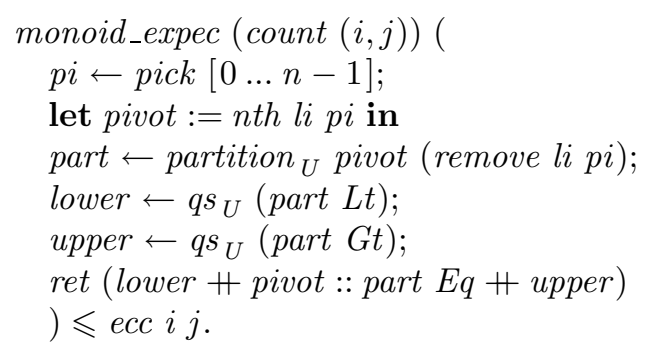

Since $c m p_{U}$ is deterministic, partition ${ }_{U}$ is as well. Furthermore, since we know exactly what monadic effects partition ${ }_{U}$ has, we can split those effects off and revert to simple effect-free filter passes. Finally, we rewrite using the following monoid_expec identity:

$$
\text { monoid_expec } f(\text { pick } l \gg m)=\operatorname{avg}(\text { map }(\text { monoid_expec } f \circ m) l) \text {. }
$$

This way, the goal ends up in a form using less monadic indirection: 


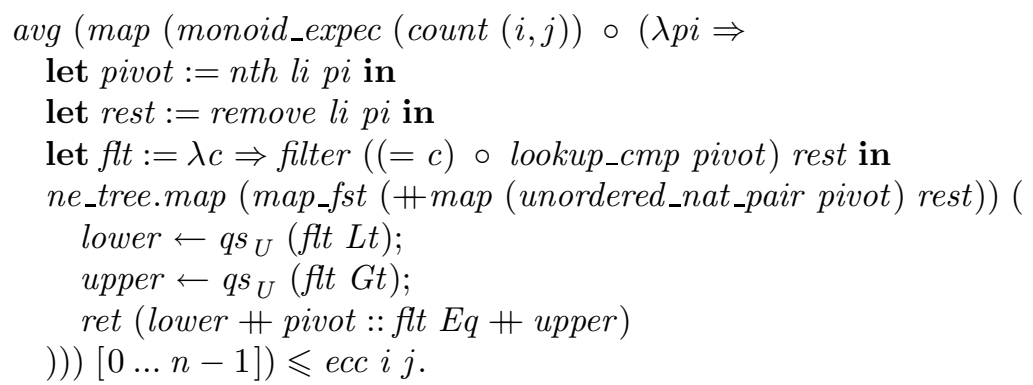

Here, map_fst applies a function to a pair's first component.

We now distinguish between five different cases that can occur for the nondeterministically picked pivot (which, because we are in the $U$ monad, is an index). It can either be less than $i$, equal to $i$, between $i$ and $j$, equal to $j$, or greater than $j$. Each case occurs a certain number of times, and has an associated expected number of $(i, j)$ comparisons (coming either from the map $f s t$ term representing the partition pass, or from the two recursive $q s_{U}$ calls). To represent this split, we first rewrite the right-hand side of the inequality to

$$
\frac{e c c i j *(i-b)+1+0+1+e c c i j *(b+n-j)}{n} .
$$

This form reflects the facts that

- the case where pivot is less than $i$ occurs $i-b$ times, and in each instance, the expected number of $(i, j)$ comparisons is no more than ecc $i j$;

- the case where the pivot is equal to $i$ occurs once, and in this case no more than a single $(i, j)$ comparison is expected;

- in the case where pivot lies between $i$ and $j$, the number of expected $(i, j)$ comparisons is 0 , and hence it does not matter how often this case occurs;

- the case where the pivot is equal to $j$ occurs once, and in this case no more than a single $(i, j)$ comparison is expected;

- the case where the pivot is greater than $j$ occurs $b+n-j$ times, and in each instance, the expected number of $(i, j)$ comparisons is no more than ecc $i j$.

With the right-hand side of the inequality in this form, we unfold the avg application on the left into sum (...) / $n$, and then cancel the division by $n$ on both sides. Next, to actually realize the split, we apply a specialized lemma stating that

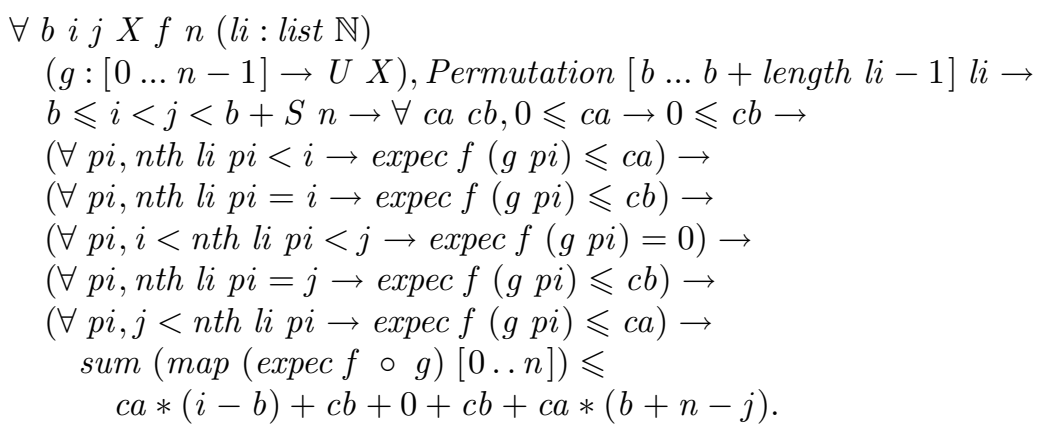


Five subgoals remain after applying this lemma - one for each listed case. The first one reads

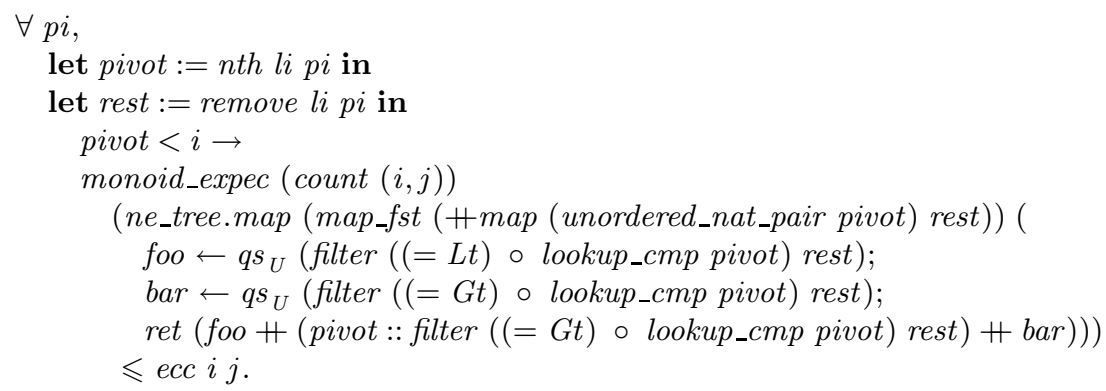

Since count $(i, j)$ is a monoid homomorphism, we may rewrite using another lemma saying that

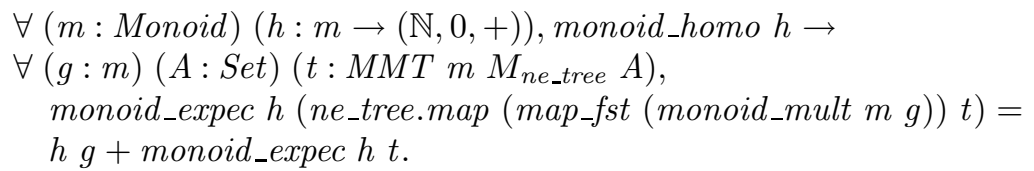

This leaves

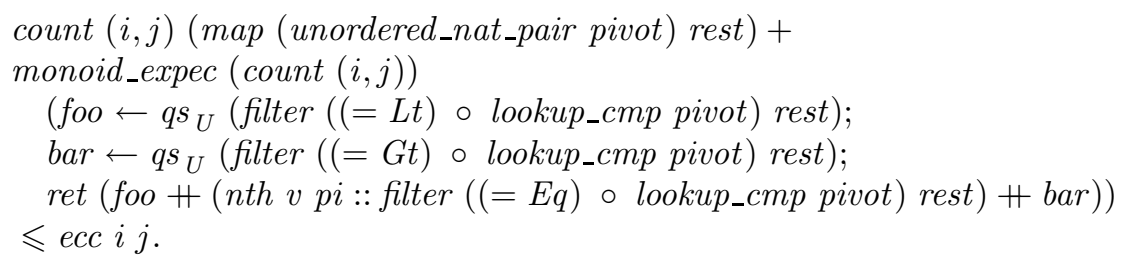

From pivot $<i$ and $i<j$, we have pivot $<j$. Since each of the comparisons in map (unordered_nat_pair pivot) rest involves the pivot element, it follows that none of them can represent comparisons between $i$ and $j$. Hence, the first term vanishes. Furthermore, monoid_expec_plus lets us distribute monoid_expec over the bind applications. Since the ret term does not produce any comparisons either (by definition), its monoid_expec term vanishes, too. What remains are the two recursive calls:

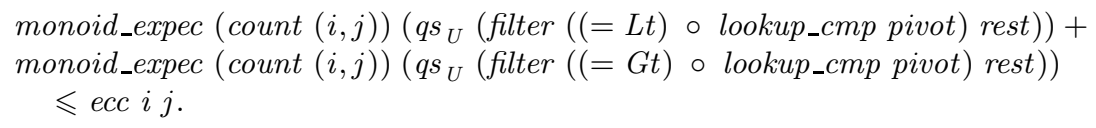

All indices in the first filtered list denote elements less than the element denoted by the pivot. Since the former precede the latter in sort $l$, it must be the case that these indices are all less than pivot. And since pivot $<i$, it follows that the first $q s_{U}$ term will produce no $(i, j)$ comparisons (using property (2) at the end of section 5 on page 267). Hence, the first monoid_expec term vanishes, leaving

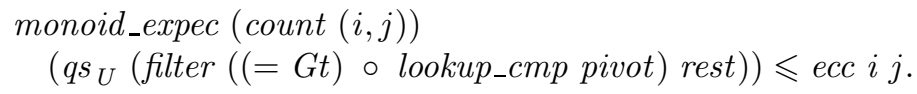


We now compare $n$th (sort $l$ ) $i$ with $n$th (sort $l$ ) pivot.

- If the two are equal, then $i$ will not occur in the filter term, and so (again) no $(i, j)$ comparisons are performed.

- If nth (sort l) $i<$ nth (sort l) pivot, then we must have $i<$ pivot, contradicting the assumption that pivot $<i$.

- If $n$th (sort l) $i>n t h$ (sort $l$ ) pivot, then we apply the induction hypothesis. For this, it must be shown that filtering the list of indices preserves contiguity, which follows from the fact that the indices share the order of the elements they denote in sort $l$.

This concludes the case where pivot $<i$. The case where $j<$ pivot is symmetric. The other three cases use similar arguments. The proof is now complete.

\section{$7 \quad$ Final Remarks}

In the interest of brevity, we have omitted lots of detail and various lemmas in the description of the proof. Still, the parts shown are reasonably faithful to the actual formalisation, with two notable exceptions.

First, we have pretended to have used ordinary natural numbers as indices into ordinary lists, completely ignoring issues of index validity that could not be ignored in the actual formalisation. There, we use vectors (lists whose size is part of their type) and bounded natural numbers in many places instead. Using these substantially reduces the amount of $i<$ length $l$ proofs that need to be produced, converted, and passed around, but this solution is still far from painless.

Second, using the Program facility to deal with Quicksort's non-structural recursion is not completely as trivial as we made it out to be. Since the recursive calls are nested in lambda abstractions passed to the bind operation of an unspecified monad, the relation between their arguments and the function's parameters is not locally known, resulting in unprovable proof obligations. To make these provable, we $\Sigma$-decorated the types of filter and partition in the actual formalisation with modest length guarantees.

The formalised development successfully adopted from the original proof the idea of using a nondeterministic version of the algorithm to make the $O(n \log n)$ bound hold for any input list, the idea of taking an order-indexed perspective to reduce the problem to a sum-of-expected-comparison-counts, and the use of the standard bound for harmonic series for the strictly numerical part. However, for the actual reduction and the derivation of the formula for the expected comparison count, the intuitive arguments essentially had to be reworked from scratch, building on the monadic representation of the algorithm and the various comparison counting/nondeterminism monads.

The shallow monadic embedding provides a simple but effective representation of the algorithm. Being parameterized on the monad used, it allows a single definition to be instantiated either with basic monads (like the identity monad or bare nondeterminism monads) to get a non-instrumented version suitable for extraction and correctness proofs, or with $M M T$-transformed monads to support 
complexity proofs. Furthermore, since this approach lets us re-use all standard Coq data types and facilities, including the powerful Program Fixpoint command, the actual algorithm definition itself is reasonably clean.

We have shown that it is straightforward to give a fully formal treatment in type theory of a classical result in complexity theory. This clearly shows the utility and applicability of the general monadic approach we have developed.

\section{References}

1. Hoare, C.: Quicksort. The Computer Journal 5, 10-15 (1962)

2. The Coq Development Team: The Coq Proof Assistant Reference Manual - Version V8.2 (February 2009), http://coq.inria.fr

3. Bertot, Y., Castéran, P.: Coq'Art: Interactive Theorem Proving and Program Development. Texts in Theoretical Computer Science. Springer, Heidelberg (2004)

4. Constable, R.L.: Expressing computational complexity in constructive type theory. In: Leivant, D. (ed.) LCC 1994. LNCS, vol. 960, pp. 131-144. Springer, Heidelberg (1995)

5. Wadler, P.: Monads for functional programming. In: Jeuring, J., Meijer, E. (eds.) AFP 1995. LNCS, vol. 925, pp. 24-52. Springer, Heidelberg (1995)

6. Sedgewick, R.: The analysis of quicksort programs. Acta Inf. 7, 327-355 (1977)

7. Sozeau, M.: Subset coercions in Coq. In: Altenkirch, T., McBride, C. (eds.) TYPES 2006. LNCS, vol. 4502, pp. 237-252. Springer, Heidelberg (2007)

8. Liang, S., Hudak, P., Jones, M.P.: Monad transformers and modular interpreters. In: POPL 1995, pp. 333-343. ACM, New York (1995)

9. Cormen, T., Leiserson, C., Rivest, R., Stein, C.: Introduction to Algorithms, 2nd edn. MIT Press, Cambridge (2001)

10. Schellekens, M.: A Modular Calculus for the Average Cost of Data Structuring. Springer, Heidelberg (2008)

11. Audebaud, P., Paulin-Mohring, C.: Proofs of Randomized Algorithms in Coq. In: Uustalu, T. (ed.) MPC 2006. LNCS, vol. 4014, pp. 49-68. Springer, Heidelberg (2006)

12. Hurd, J.: Formal Verification of Probabilistic Algorithms. PhD thesis, University of Cambridge (2002) 